\title{
Observations of Extragalactic Objects with the Hawaii Imaging Fabry-Perot Interferometer (HIFI)
}

\author{
R. Brent Tully \\ Institute for Astronomy, University of Hawaii
}

\begin{abstract}
Imaging Fabry-Perot interferometers have a particularly useful role to play in studies of the extended regions of anomalous emission in active galaxies. It becomes possible to study the velocity and excitation structure of warm gas with full coverage of large fields. Observations and modeling of several good examples of transient activity will be described as a way of demonstrating the power of an operational facility.
\end{abstract}

\section{Introduction}

HIFI is more a collaboration and an approach to a research program than a specific piece of equipment. It involves the use of Fabry-Perot interferometers on the University of Hawaii $2.2 \mathrm{~m}$ and Canada-France-Hawaii $3.6 \mathrm{~m}$ telescopes with a variety of $C C D$ detectors and a suite of reduction programs developed in a UNIX environment. At the Canada-France-Hawaii telescope, the facility focal reducers PALILA or MOS/FP are used with either facility or our personal etalons and filters. At the University of Hawaii telescope, there is an optical bench that affords a variety of focal reductions and etalon choices. In all cases, the Fabry-Perot interferometers are piezoelectric gap control systems purchased from Queensgate Instruments.

This short review will draw attention to the convolution of technological and astrophysical drivers that has motivated the direction taken by the HIFI collaboration. To begin, there are the usual three fundamental advantages of the imaging Fabry-Perot system: (i) spectral coverage of a two-dimensional field, (ii) free choice of spectral resolution, and (iii) sensitivity to faint emission. Initially it was the first point, the possibility to have 2-D kinematic coverage, that most interested us. This advantage is greatest where velocity fields are most complex and extensive (ie, least adequately sampled by long slits). We turned our attention to the extended regions of anomalous emission around active galactic nuclei over a decade ago, when little was known about them except that the velocity fields were complicated.

With the way that Fabry-Perots were being used in the past, active nuclei presented a problem because there can be large velocity excursions, hence interorder confusion and even blending of adjacent spectral lines. For our work it is essential to isolate a single interference order. Simultaneously, we want to retain adequate spectral resolution. The solution is to go for a large free-spectral-range by working in a low order and to recover spectral resolution by working at as high a finesse as possible. Typical parameters for our operation are spectral res- 
olution of $35 \mathrm{~km} / \mathrm{s}$ and free-spectral-range of $2500 \mathrm{~km} / \mathrm{s}$, achieved with a finesse of 70 .

We soon appreciated that this single-order mode of operation has a second advantage. It gives us the capability to achieve precision spectrophotometry. There are some requirements: (i) a single order must be isolated with a blocking filter with a width of no more than half the free-spectral-range between orders, (ii) a CCD detector is used because it is linear and mechanically stable, and (iii) extensive and high signal-to-noise white light, calibration lamp, and standard star data cubes must be obtained. The subject of spectrophotometry with a Fabry-Perot is discussed by Bland-Hawthorn in this same proceedings. See also Bland and Tully (1989) for a general discussion of HIFI and our reduction procedures.

A few additional comments on the way we do business are the following. In order to minimize problems with ghost images we tilt the Fabry.Perot plates so the interference pattern is offset to the edge of the field, we place the order blocking filter in the converging beam, and we are sure to use a field stop. Since the start, we have used a CCD detector and now with read noise at or below 5 electrons we are essentially in the photon counting regime with integrations of 5 minutes or more. Interleaved scans minimize problems with changing observing conditions (not a major problem at Mauna Kea). Drifts due to lack of temperature stabilization are a minor concern (something we should improve) but it is often possible to self-calibrate the spectral zero-point on frames from airglow emission. It is not necessary to scan a full free-spectral-range but only the part of that range that covers the spectral region of interest. The scan range can be minimized by judicious orientation of the ring center with respect to the field. Ideally, the ring center should be on the redshifted side of the field. The final data cube should be Nyquist sampled, that is, adjacent spectral frames are separated by half the width of the Airy profile at half maximum intensity. Care should be taken to extend the scan sufficiently to define the profile baseline.

White light exposures of the dome are taken at high signal-to-noise at essentially every gap setting with each blocking filter. This process takes several hours and is done the day following the observations on the sky. Absolute flux calibrations are obtained with a small number of etalon settings on spectrophotometric standards, preferably very hot stars with negligible Balmer features.

\section{Discussion of a Few Results}

There is a panoply of physical mechanisms that can interplay to disrupt galaxies from equilibrium conditions. These mechanisms can have different relative importance from one case to another, total energetics may differ, and we observe events as snapshots in time and viewing perspective. No one case study, or even a small number of cases, can provide a clear picture of galaxy disruptions. However, as different examples are studied, recurrent patterns emerge. Certain physical mechanisms can be isolated, in some cases better than in others. One can imagine that circumstances between two cases differ in age, or line-of-sight obscuration, or beam alignment, or whatever. Through the process of looking at many examples, reasonably coherent scenarios can be formulated for what can 
happen and these conjectures can be tested by looking at further cases. A few intruiging subjects will be described here as examples of the discovery process and the possibilities.

This article is an advertisement for Fabry-Perot applications rather than a review of peculiar galaxiss. Everything that will be discussed has been presented in greater detail in other places. The only references provided are to the original Fabry-Perot studies. Those sources provide references to other works.

\section{1. $\quad$ M 82}

This galaxy is one of our nearest neighbors and, as such, it can be be studied with high resolution. Images restricted to the wavelengths of optical recombination emission lines give the impression that the galaxy is being blown apart, with filaments that emanate from the central $500 \mathrm{pc}$ region sprayed across the full dimensions of the system. The galaxy is an abnormally luminous source of far-infrared radiation and has been identified as a system undergoing an intense burst of star formation at the present time. Discrete sources of non-thermal radio emission in the central region are thought to arise from the remnants of large numbers of supernovae, the product of the most massive progeny of the starburst.

In spite of the apparent chaos in the filamentary recombination line structures, there is considerable order in the velocity field. Consequently, this galaxy is something of a Rosetta Stone for the study of explosive events. The line profiles in the filaments that emerge toward the poles of the galactic plane are systematically doubled. From the pattern, with doubling greatest along the galaxy minor axis and blending at the edges of the emission cones, it is evident that we are observing emission on surfaces of bubbles. X-ray observations demonstrate that there is $10^{7} \mathrm{~K}$ gas within the bubbles. At optical wavelengths we observe the cool sheaths on the surfaces of hot bubbles, where thermal cooling instabilities have led to filamentary fragmentation.

The Fabry-Perot spectroscopic information further tells us that velocities are in the sense of outflow and increase monotonically from the center of the galaxy. They attain an amplitude that assures that the gas can excape from the potential well of the system.

There is enough information from the X-ray, optical, infrared, and radio data to piece together a cohesive picture of what has happened in M 82. A massive starburst was initiated, probably by the infusion of gas toward the center by the close passage of the companion M 81. The subsequent multitude of supernovae dumped energy into the interstellar medium. This thermalized hot gas tried to expand but was constricted in the plane where there is a high density of cold gas and instead pushed out at the poles of the disk. Hence a galactic wind developed, though restricted to polar cones. Once above a dynamic scale-height, the energy density and decreasing gravity lead to outward acceleration of the plasma. The gas piles up around the edges and is cool enough that optical recombination radiation is emitted.

The general picture that has emerged from the HIFI observations of M 82 were presented by Bland and Tully (1988). A more detailed analysis of the same data is being undertaken by Shopbell (1994). The galaxy NGC 3079 discussed by 
Veilleux at this conference is another, and even more energetic, example of a galactic wind in an active galaxy.

\subsection{NGC 1068}

This Seyfert galaxy is rich in symptoms of anomalous behaviour, which is to say it is a confusing situation. However there is some order amid the confusion. Within $10^{\prime \prime}$ of the nucleus, the Fabry-Perot spectra can be decomposed into three physical components. Ten percent of the flux at H $\alpha$ has FWHM $\sim 600$ $\mathrm{km} / \mathrm{s}$ and is associated with the barely resolved Seyfert 2 nucleus. Five percent of the flux is associated with truly narrow lines, with FWHM $\sim 100 \mathrm{~km} / \mathrm{s}$, which arises out of ambient gas that is piled up on the boundaries of the radio lobe 'working surfaces'. The remaining $85 \%$ of the flux has high redshifted and blueshifted velocities and is closely associated with the radio continuum lobes observed with the Very Large Array. In particular, there is profile doubling of this latter component at symmetric locations at the north-east and south-west bases of the radio jet 'working surfaces'.

In addition to the morphological correspondences with the VLA non-thermal radio maps already noted, we can draw on X-ray, optical polarization, and high spatial resolution Space Telescope observations to develop a model of what is happening near the center of NGC 1068. There is two-sided ejection of relativistic plasma which creates the radio jets and which terminates at the 'working surface' shock fronts. There is a thermalized wind component in fans of rather wide opening angles $\left(\sim 80^{\circ}\right)$ which gives rise to X-ray emission. With HIFI, we see the cooler gas that encases the jets and entrained winds. We see shocked gas associated with cooling instabilities on the boundaries of the wind and jet and shocked ambient gas with narrower lines. In each case, the characteristic $[\mathrm{NII}] / \mathrm{H} \alpha$ line intensity ratios of high excitation gas is observed. The HIFI and Space Telescope information are consistent with the proposition that the cone of the outflow wind is tilted with respect to the plane of the galaxy but does not point toward the poles. Our relatively face-on viewing angle to the disk is not within the frontside cone and hence we do not see the Seyfert broad line region directly. However, it is inferred to exist from broad line emission seen in reflection.

The influence of the active nucleus is seen to much larger distances in the disk of the galaxy. Out to several kiloparsecs, the diffuse interstellar gas has high excitation conditions marked by strong and relatively broad [NII] and [OIII]. This emission is particularly pronounced within projections of the outflow cones identified in the nuclear regions. It is concluded that gas at large radii have a direct view of the Seyfert 1 nucleus and this gas is being photoionized by a hard uv spectrum.

While the nucleus is affecting the outer disk of the galaxy, probably phenomena in the disk are also affecting the nucleus. There is the clear kinematic signature of bar streaming in the HIFI velocity field. The associated transfer of angular momentum and mass probably accounts for the starburst in an annulus at 900 pc. It can be speculated that the inflow of material propagates down to the scale of the active nucleus and provides fuel, though the processes involved are not clarified.

The HIFI contributions to the study of NGC 1068 are reported by Cecil, Bland, 
and Tully (1990), Bland-Hawthorn, Sokolowski, and Cecil (1991), and Sokolowski, Bland-Hawthorn, and Cecil (1991). Reports on the large-scale kinematic features are in preparation by Bland-Hawthorn et al.

\section{3. $\mathbf{M} 51$}

The nucleus of M 51 displays a mild form of activity within $10^{\prime \prime}$ of the center. A particularly noteworthy point is the marked similarity between the non-thermal VLA maps and the high excitation [NII] line emission map. The VLA data indicate that there is two-sided ejection from the nucleus. The activity on the two sides may not date from the same epoc because on one side there is a bright thin jet terminating in a working surface while on the other side the synchrotron emission is from a diffuse, more extended bubble. The optical emission has a very similar morphology. The highest velocity features are associated with the radio jet 'working surface'.

The model we propose for this system holds that the line emission arises from shocked gas in the sheath around the relativistic plasma in the radio jets. Discrete line profile components can be associated with shocks traveling away from the working surface at the inferred sound speed in the interstellar medium. At localized points in the working surface shock, the ratio of [NII] $\lambda 6583$ to $\mathrm{H} \alpha$ reaches as high as 5 . It is difficult to explain such a high ratio if the gas is in equilibrium. The HIFI report on $\mathrm{M} 51$ is by Cecil (1988).

\subsection{NGC 3516}

This Seyfert galaxy offers another partial example of a good correspondence between radio non-thermal maps and the morphology of high-excitation optical line emission. Partial, in the sense that there is a one-armed radio jet but a two-armed corresponding optical structure. We see the approaching feature at radio wavelengths but not the receding one. The structures are curved and the highest velocities in the optical structures are at the bases of the jets near the nucleus.

In the case of NGC 3516 we were able to invent two alternative models that describe the observations. It is a warning example of the non-uniqueness of models and challenges us to be discriminating. With one model, the bi-symmetric jets are curved because of precession. Motions are low at large radii and high near the center because the precession is bringing the jets from the plane of the sky toward our line of sight. With the second model, the jets are in the plane of the disk of the galaxy and the outflowing material is bent by collisions with ambient gas. Details are provided by Veilleux, Tully, and Bland-Hawthorn (1993).

\subsection{NGC 4258}

This final example is described in more detail by Cecil at this conference and in the literature by Cecil, Wilson, and Tully (1992). The bi-symmetric anomalous arms that extend across kiloparsec scales were discovered by our host (Courtès and Cruvellier 1961). Today we refer to them as jets. They are detected at radio, optical recombination, and X-ray wavelengths. Material is being ejected from the nucleus of NGC 4258 and is traveling in thin, curved filaments across the dimensions of the galaxy. At some places there is good evidence that the jets 
impinge and are deflected by ambient clouds of gas. Perhaps the most dramatic, but still uncertain proposition in connection with these jets is that they are composed of several separate strands that are helically coiled. The jets in this galaxy may be a laboratory for studies of streaming instabilities and magnetic confinement.

\section{Summary}

As of the present, the HIFI collaboration has observed some 18 different extragalactic targets. In addition to the kinds of galaxies that are mentioned in this article, we have observed ultraluminous far-infrared objects, cooling flow clusters, emission-line ellipticals, and potential intergalactic sources. There is both diversity and commonality. For example, galactic winds can arise either from intense bursts of star formation or from the energy output of active nuclei. Outflow can be well-collimated or may be only poorly constrained to wide-angle bubbles.

There are still so many issues that are only partially understood. While there are almost always signs of bi-polar ejection, sometimes the two sides show different manifestations which hint at different stages of development (ages?). Outflows are commonly toward the poles of the galaxy disks but definitely there are exceptions. When jets are bent, is it because of precession or deflection? Often we are confident that the optical emission being observed is excited in shocks but there are instances where the gas is probably excited by photoionization from sources of hard radiation. Then, in studying the interacting/starburst systems and cooling flows and barred galaxies with streaming motions we might learn about the mechanisms that regulate gas inflow, a prerequisite for the ignition of the explosive outflows.

Acknowledgments. The HIFI team consists of Joss Bland-Hawthorn, Gerald Cecil, Jon Morse, Patrick Shopbell, Jim Sokolowski, Sylvain Veilleux, and the author. Andrew Wilson has participated on several occasions. We have depended on facility equipment at the Canada-France-Hawaii, University of Hawaii, and Cerro Tololo telescopes. Funding has been provided by grants from the US National Science Foundation.

\section{References}

Bland, J. and Tully, R.B. 1988, Nature, 334, 43.

Bland, J. and Tully, R.B. 1989, AJ, 98, 723.

Bland-Hawthorn, J., Sokolowski, J., and Cecil, G.N. 1991, ApJ, 375, 78.

Cecil, G.N. 1988, ApJ, 329, 38.

Cecil, G.N., Bland, J., and Tully, R.B. 1990, ApJ, 355, 70.

Cecil, G.N., Wilson, A.S., and Tully, R.B. 1992, ApJ, 390, 365

Courtès, G., and Cruvellier, P. 1961, C. r. Acad. Sci. Paris, 253, 218.

Shopbell, P. 1994, Ph.D. Dissertation, Rice University.

Sokolowski, J., Bland-Hawthorn, J., and Cecil, G.N. 1991, ApJ, 375, 583.

Veilleux, S., Tully, R.B., and Bland-Hawthorn, J. 1993, AJ, 105, 1318. 